\title{
UNSTEADY THREE-DIMENSIONAL MHD NANOFLUID FLOW OVER A STRETCHING SHEET WITH VARIABLE WALL THICKNESS AND SLIP EFFECTS
}

\author{
G. VINOD KUMAR*, S.V.K. VARMA and R.V.M.S.S.K. KUMAR \\ Department of Mathematics, S.V. University \\ Tirupati-517502, A.P, INDIA \\ E-mails: gvkphd@gmail.com; svijayakumarvarma@yahoo.co.in; kksaisiva@gmail.com
}

\begin{abstract}
The stretching sheets with variable thickness may occur in engineering applications more frequently than a flat sheet. Due to its various applications, in the present analysis we considered a three dimensional unsteady MHD nanofluid flow over a stretching sheet with a variable wall thickness in a porous medium. The effects of radiation, viscous dissipation and slip boundary conditions are considered. Buongiorno's model is incorporated to study the combined effects of thermophoresis and Brownian motion. The dimensionless governing equations are solved by using MATLAB bvp4c package. The impact of various important flow parameters is presented and analysed through graphs and tables. It is interesting to note that all the three boundary layer thicknesses are diminished by slip parameters. Further, the unsteady parameter decreases the hydromagnetic boundary layer thickness.
\end{abstract}

Key words: unsteady flow, magnetic field, Buongiorno's model, variable wall thickness sheet, velocity, thermal and solutal slip.

\section{Introduction}

The study of fluid flow in a stretched boundary layer accompanied by heat transfer has received great interest of scientists due to the applications which are widely spread in many areas. The heating and cooling process in heat transfer analysis plays an important role in various industries such as manufacturing, electronics and transportation, etc. During this process metals are heated and cooled in a specific order so that to keep the metal away from molten state. The steady two dimensional boundary layer flow of a Newtonian fluid over a stretching surface has been studied by Crane [1]. The boundary layer flow and heat transfer of nanofluids over a stretching sheet is a vital concept in the present research era. These concepts provide many applications over a broad spectrum of science and engineering disciplines. The heat transfer feature is an important aspect in the study of boundary layer flow of a nanofluid over a stretching sheet. The boundary layer flow of a nanofluid over a stretching sheet was studied by Khan and Pop [2]. They found that the Brownian motion and thermophoresis parameters cause a reduction in the heat transfer rate. Nadeem and Lee [3] analysed the impact of a nanofluid over an exponential stretching sheet. Mansur and Ishak [4] discussed the unsteady nanofluid flow over a stretching/shrinking sheet with convective boundary condition. The illustrative studies on boundary layer flow of a nanofluid over a stretching sheet can be found in [5-8].

The no-slip boundary condition is often utilized in the flow problems of viscous fluids. However, there are numerous cases where such condition is inadequate and slip may occur on the boundary, when the fluid is particulate such as emulsions, suspensions, foams and polymer solutions. The behaviour of a fluid flow subject to the slip flow governance shows great discrepancy from the traditional flow. The discrepancy between the fluid velocity at the wall and the velocity of the wall itself is directly proportional to the shear stress. The proportionality constant is called the slip length (Maxwell [9], Hak [10]). Beavers and Joseph

\footnotetext{
* To whom correspondence should be addressed
} 
[11] investigated fluid flow over a permeable wall with a slip boundary condition. The boundary layer flow of a nanofluid over non-linear permeable stretching sheet at prescribed surface temperature was discussed by Das [12]. Goyal and Bhargava [13] explained the visco-elastic nanofluid flow past a stretching sheet with partial slip condition. They found that the visco-elastic parameter contraverses the direction of fluid flow when it is close to the stretching sheet and far away from the sheet. Awais et al. [14] investigated the MHD stagnation point flow of a nanofluid over a stretching sheet with slip boundary conditions. Raza et al. [15] examined the hydromagnetic boundary layer flow of a nanofluid in a rotating channel with the slip conditions and suction/injection. The study of nanofluid with slip conditions over a stretching sheet was carried out by the authors [16-19].

The stretching sheet with variable thickness has more engineering applications than a flat sheet. So, many authors have concentrated on variable thickness sheets. Among them, Fang et al. [20] considered the boundary layer flow over a stretching sheet with variable wall thickness and observed that the boundary layer becomes thinner for $m<1$ and becomes thicker for $m>1$. Khader and Ahmed [21] presented the numerical solutions for the steady boundary layer slip flow over a stretching sheet with variable wall thickness. Anjali Devi and Prakash [22, 23] studied the effects of thermal conductivity, temperature dependent viscosity and radiation on MHD slendering sheet. Abdel-wahed et al. [24] presented the nanofluid flow over a moving surface with non-linear velocity and variable wall thickness under the influence of a magnetic field and heat source. Kumar and Varma [25] investigated the magnetohydrodynamic nanofluid flow through a porous medium over a slendering sheet under the influence of viscous dissipation, thermal radiation and chemical reaction and found that the radiation parameter, thermophoresis parameter enhance the nanofluid temperature. Later, Kumar et al. [26] analysed the hydromagnetic 3D slip flow over a slendering sheet by using single walled and multi-walled nanotubes. Hayat et al. [27] examined the hydromagnetic flow of a tangent hyperbolic nanofluid over a stretching sheet with variable wall thickness. Acharya et al. [28] discussed the MHD slendering stretched flow by using $\mathrm{TiO}_{2}$ and $\mathrm{Ag}$ nanoparticles with water as a base fluid. Very recently, Prasad et al. [29] studied hydromagnetic flow of a nanofluid over a slender elastic sheet with variable wall thickness, thermal conductivity and species molecular diffusion.

The main goal of this article is to investigate a three dimensional unsteady flow of a hydromagnetic nanofluid over a stretching sheet with variable wall thickness in a porous medium. Thermal radiation and viscous dissipation effects are taken into account. The resulting set of ordinary differential equations is solved by using MATLAB bvp4c package. The influence of various important flow parameters on the velocity, temperature, nanofluid concentration as well as the friction factor coefficient and the rate of heat and mass transfer coefficients are analysed through graphs and tables.

\section{Formulation of the problem}

A three-dimensional hydromagnetic nanofluid flow over a stretching sheet with variable wall thickness in a porous medium has been considered in the present work. The velocity, thermal and solutal slip conditions, viscous dissipation and thermal radiation effects are taken into account. Buongiorno's model is incorporated. The $x$-axis is chosen in the direction of flow, $y$ and $z$ axes are perpendicular to it (see

Fig.1). Let $u_{w}(x, y, t)=\frac{U_{0}(x+y+c)^{n}}{(1-\lambda t)}$ and $v_{w}(x, y, t)=\frac{U_{0}(x+y+c)^{n}}{(1-\lambda t)}$ be the stretching sheet velocities along the $x$ and $y$ directions, respectively. We assume that the sheet is not flat but rather is defined as $z=E(x+y+c)^{\frac{1-n}{2}}(n \neq 1)$. The coefficient $E$ is chosen as a small constant so that the sheet is sufficiently thin to avoid a measurable pressure gradient along the sheet. A variable magnetic field $B(x, y, t)=B_{0}\left(\frac{(x+y+c)^{\frac{n-1}{2}}}{\sqrt{1-\lambda t}}\right)$ is applied normal to the flow direction. The non-uniform permeability of the 
porous medium can be taken in the form $k_{0}=k_{1}\left(\frac{1-\lambda t}{(x+y+c)^{n-1}}\right)$. The thickness of the sheet may decrease or increase with distance from the slot, which is dependent on $n$. For $n=1$, the sheet is flat.

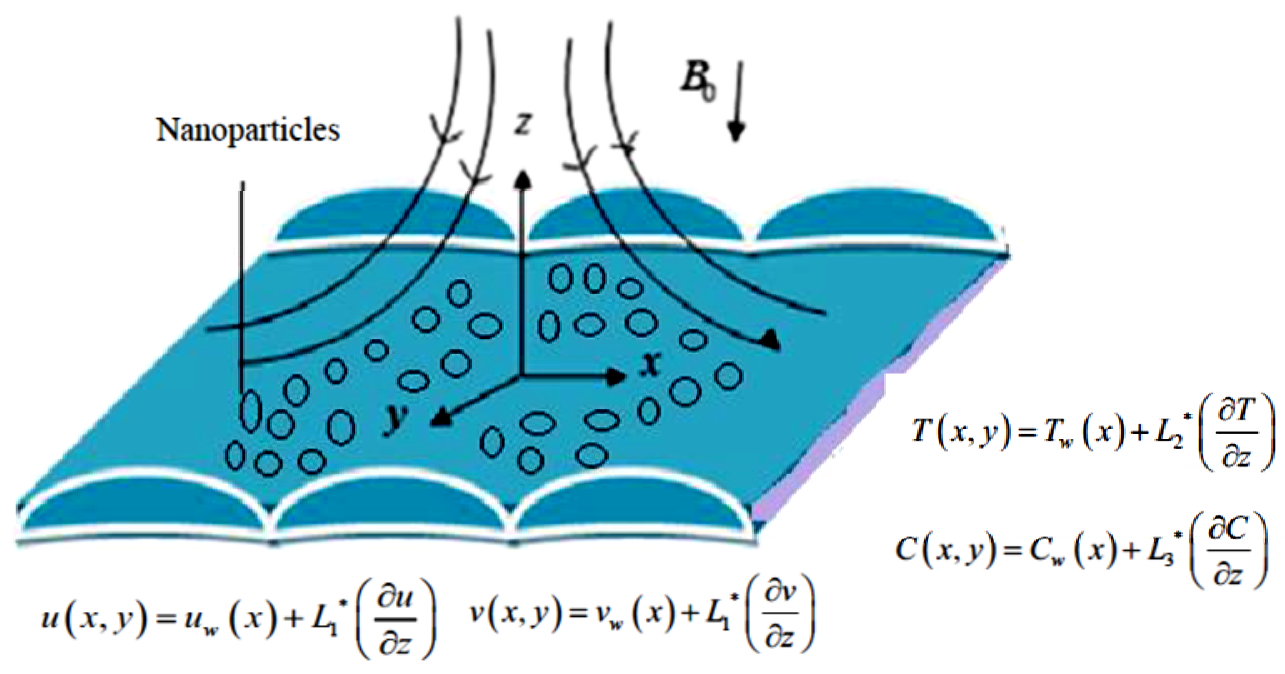

Fig.1 The physical model of flow configuration.

Under the aforesaid assumptions the governing boundary layer equations for an unsteady three dimensional flow is

$$
\begin{aligned}
& \frac{\partial u}{\partial x}+\frac{\partial v}{\partial y}+\frac{\partial w}{\partial z}=0, \\
& \left(\frac{\partial u}{\partial t}+u \frac{\partial u}{\partial x}+v \frac{\partial u}{\partial y}+w \frac{\partial u}{\partial z}\right)=v \frac{\partial^{2} u}{\partial z^{2}}-\frac{\sigma B^{2}(x, y, t)}{\rho_{f}} u-\frac{v}{k_{0}} u, \\
& \left(\frac{\partial v}{\partial t}+u \frac{\partial v}{\partial x}+v \frac{\partial v}{\partial y}+w \frac{\partial v}{\partial z}\right)=v \frac{\partial^{2} v}{\partial z^{2}}-\frac{\sigma B^{2}(x, y, t)}{\rho_{f}} v-\frac{v}{k_{0}} v, \\
& \left(\frac{\partial T}{\partial t}+u \frac{\partial T}{\partial x}+v \frac{\partial T}{\partial y}+w \frac{\partial T}{\partial z}\right)=\alpha \frac{\partial^{2} T}{\partial z^{2}}+\tau\left(D_{B} \frac{\partial C}{\partial z} \frac{\partial T}{\partial z}+\frac{D_{B}}{T_{\infty}}\left(\frac{\partial T}{\partial z}\right)^{2}\right)+ \\
& \frac{1}{\rho C_{p}} \frac{\partial q_{r}}{\partial z}+\frac{v}{C_{p}}\left(\left(\frac{\partial u}{\partial z}\right)^{2}+\left(\frac{\partial v}{\partial z}\right)^{2}\right), \\
& \frac{\partial C}{\partial t}+u \frac{\partial C}{\partial x}+v \frac{\partial C}{\partial y}+w \frac{\partial C}{\partial z}=D_{B} \frac{\partial^{2} C}{\partial z^{2}}+\frac{D_{T}}{T_{\infty}} \frac{\partial^{2} T}{\partial z^{2}} .
\end{aligned}
$$

The appropriate boundary conditions are 


$$
\begin{array}{ll}
u=u_{w}(x)+L_{1}^{*}\left(\frac{\partial u}{\partial z}\right), & v=v_{w}(x)+L_{1}^{*}\left(\frac{\partial v}{\partial z}\right), \quad w=0, \\
T=T_{w}+L_{2}^{*}\left(\frac{\partial T}{\partial z}\right), & C=C_{w}+L_{3}^{*}\left(\frac{\partial C}{\partial z}\right),
\end{array}
$$

and

$$
u=0, \quad v=0, \quad T \rightarrow T_{\infty}, \quad C \rightarrow C_{\infty} \quad \text { as } \quad z \rightarrow \infty,
$$

where

$$
\begin{aligned}
& L_{1}^{*}=\left[\frac{2-f_{1}}{f_{1}}\right] \xi_{1}\left(\sqrt{\frac{(x+y+c)^{1-n}}{1-\lambda t}}\right), \quad \xi_{2}=\left(\frac{2 \gamma}{\gamma+1}\right) \frac{\xi_{1}}{\operatorname{Pr}}, \\
& L_{2}^{*}=\left[\frac{2-b}{b}\right] \xi_{2}\left(\sqrt{\frac{(x+y+c)^{(1-n)}}{1-\lambda t}}\right), \quad \xi_{3}=\left(\frac{2 \gamma}{\gamma+1}\right) \frac{\xi_{2}}{\operatorname{Pr}}, \\
& L_{3}^{*}=\left[\frac{2-d}{d}\right] \xi_{3}\left(\sqrt{\frac{(x+y+c)^{(1-n)}}{1-\lambda t}}\right), \\
& \left.T_{w}(x, y, t)=T_{\infty}+T_{0} \sqrt{\frac{(x+y+c)}{1-\lambda t}}, \quad C_{w}(x, y, t)=C_{\infty}+C_{0} \sqrt{\frac{(x+y+c)^{(1-n)}}{1-\lambda t}}\right\} \text { for } n \neq 1 .
\end{aligned}
$$

The Roseland approximation is taken as

$$
q_{r}=-\frac{4 \sigma^{*}}{3 k^{*}} \frac{\partial T^{4}}{\partial z}
$$

where $k^{*}$ is the mean absorption coefficient, $\sigma^{*}$ is the Stefan-Boltzman constant and the linear temperature $T^{4}$ is expanded by using the Taylor series in terms of $T_{\infty}$ as

$$
T^{4}=4 T_{\infty}^{3} T-3 T_{\infty}^{4} .
$$

In view of Eqs (2.11) and (2.12), Eq.(2.4) can be written as

$$
\begin{aligned}
& \left(\frac{\partial T}{\partial t}+u \frac{\partial T}{\partial x}+v \frac{\partial T}{\partial y}+w \frac{\partial T}{\partial z}\right)=\alpha \frac{\partial^{2} T}{\partial z^{2}}+\tau\left(D_{B} \frac{\partial C}{\partial z} \frac{\partial T}{\partial z}+\frac{D_{B}}{T_{\infty}}\left(\frac{\partial T}{\partial z}\right)^{2}\right)+ \\
& \frac{16 \sigma^{*} T_{\infty}^{3}}{3\left(\rho C_{p}\right) k^{*}} \frac{\partial^{2} T}{\partial z^{2}}+\frac{v}{C_{p}}\left(\left(\frac{\partial u}{\partial z}\right)^{2}+\left(\frac{\partial v}{\partial z}\right)^{2}\right) .
\end{aligned}
$$

\section{Solution of the problem}

The above mentioned dimensional equations are transformed to dimensionless form by using the following suitable similarity transformations 


$$
\begin{aligned}
& \eta=z\left(\frac{(n+1) U_{0}}{2 v}\right)^{0.5}\left(\frac{(x+y+c)^{(n-1) 0.5}}{\sqrt{1-\lambda t}}\right), \quad u=\frac{U_{0}(x+y+c)^{n}}{1-\lambda t} \frac{\partial f}{\partial \eta}, \quad v=\frac{U_{0}(x+y+c)^{n}}{1-\lambda t} \frac{\partial g}{\partial \eta}, \\
& w=-\left(\frac{2 U_{0} v}{n+1}\right)^{0.5}\left(\frac{(x+y+c)^{(n-1) 0.5}}{\sqrt{1-\lambda t}}\right)\left[\frac{n+1}{2}(f+g)+\eta\left(\frac{n-1}{2}\right)\left(\frac{\partial f}{\partial \eta}+\frac{\partial g}{\partial \eta}\right)\right] \\
& T=T_{\infty}+\left(T_{w}-T_{\infty}\right) \theta, \quad C=C_{\infty}+\left(C_{w}-C_{\infty}\right) \phi .
\end{aligned}
$$

In view of the above similarity transformations the set of Eqs (2.2), (2.3), (2.5) and (2.13) and the boundary conditions (2.6) are transformed as

$$
\begin{aligned}
& f^{\prime \prime \prime}-\left(\frac{2 n}{n+1}\left(f^{\prime}\right)^{2}+\frac{2 n}{n+1} f^{\prime} g^{\prime}-(f+g) f^{\prime \prime}\right)-(M+K) f^{\prime}-\frac{2 A}{n+1} f^{\prime}=0, \\
& g^{\prime \prime \prime}-\left(\frac{2 n}{n+1}\left(g^{\prime}\right)^{2}+\frac{2 n}{n+1} f^{\prime} g^{\prime}-(f+g) g^{\prime \prime}\right)-(M+K) g^{\prime}-\frac{2 A}{n+1} g^{\prime}=0 \\
& \frac{1}{\operatorname{Pr}}\left[1+\frac{4 R}{3}\right] \theta^{\prime \prime}+(f+g) \theta^{\prime}+\mathrm{Ec}_{x}\left(f^{\prime \prime}\right)^{2}+\mathrm{Ec}_{y}\left(g^{\prime \prime}\right)^{2}-\frac{A \eta}{n+1} \theta^{\prime}+N b \theta^{\prime} \phi^{\prime}+N t\left(\theta^{\prime}\right)^{2}=0 \\
& \phi^{\prime \prime}+\frac{N t}{N b} \theta^{\prime \prime}-\frac{A \eta}{n+1} \phi^{\prime} \mathrm{Sc}+\operatorname{Sc}(f+g) \phi^{\prime}=0
\end{aligned}
$$

and the corresponding boundary conditions are

$$
\begin{aligned}
& f(0)=\alpha\left(\frac{1-n}{n+1}\right)\left[1+\left.L_{1}\left(f^{\prime \prime}\right)\right|_{\eta=0}\right], \quad f^{\prime}(0)=\left[1+\left.L_{l}\left(f^{\prime \prime}\right)\right|_{\eta=0}\right], \\
& g(0)=\alpha\left(\frac{1-n}{n+1}\right)\left[1+\left.L_{1}\left(g^{\prime \prime}\right)\right|_{\eta=0}\right], \quad g^{\prime}(0)=\left[1+\left.L_{l}\left(g^{\prime \prime}\right)\right|_{\eta=0}\right], \\
& \theta(0)=\left[1+\left.L_{2}\left(\theta^{\prime}\right)\right|_{\eta=0}\right], \quad \phi(0)=\left[1+\left.L_{3} \phi^{\prime}\right|_{\eta=0}\right], \\
& f^{\prime}=0, \quad g^{\prime}=0, \quad \theta=0, \quad \phi=0 \quad \text { as } \quad \eta \rightarrow \infty
\end{aligned}
$$

where $L_{1}=\left(\frac{2-f_{1}}{f_{1}}\right) \xi_{1} \sqrt{\frac{U_{0}(n+1)}{2 v}}, L_{2}=\left(\frac{2-b}{b}\right) \xi_{2} \sqrt{\frac{U_{0}(n+1)}{2 v}}, L_{3}=\left(\frac{2-d}{d}\right) \xi_{3} \sqrt{\frac{U_{0}(n+1)}{2 v}}$, $M=\frac{2 \sigma B_{0}^{2}}{\rho_{f} U_{0}(n+1)}, \operatorname{Pr}=\frac{\mu C_{p}}{k}, N b=\frac{\tau D_{B}\left(C_{w}-C_{\infty}\right)}{v}, N t=\frac{\tau D_{B}\left(T_{w}-T_{\infty}\right)}{v T_{\infty}}, \mathrm{Sc}=\frac{v}{D_{B}}, K=\frac{2 v}{U_{0}(n+1) k_{1}}$, $A=\frac{\lambda}{U_{0}}(x+y+c)^{l-n}, R=\frac{4 \sigma^{*} T_{\infty}^{3}}{k k^{*}}, \mathrm{Ec}_{x}=\frac{u_{w}^{2}}{c_{p}\left(T_{w}-T_{\infty}\right)}, \mathrm{Ec}_{y}=\frac{v_{w}^{2}}{c_{p}\left(T_{w}-T_{\infty}\right)}$ and $\alpha=E \sqrt{\frac{U_{0}(n+1)}{2 v}}$.

The skin friction coefficient, heat and mass transfer rates are defined as 


$$
\begin{aligned}
& C f_{x}(\operatorname{Re})^{\frac{1}{2}}=\left.2\left(\frac{n+1}{2}\right)^{0.5}\left(f^{\prime \prime}\right)\right|_{\eta=0}, \quad \mathrm{Nu}_{x}(\operatorname{Re})^{-\frac{1}{2}}=-\left.\left(\frac{n+1}{2}\right)^{0.5}\left(\theta^{\prime}\right)\right|_{\eta=0}, \\
& S h_{x}=-\left.\left(\frac{n+1}{2}\right)^{0.5}(\operatorname{Re})^{\frac{1}{2}}\left(\phi^{\prime}\right)\right|_{\eta=0} .
\end{aligned}
$$

Here $\operatorname{Re}_{x}=\frac{u_{w}(x+y+c)}{v}$ is the local Reynolds number.

\section{Results and discussion}

The set of ordinary differential Eqs (3.3)-(3.5) with the corresponding boundary conditions (3.6) is solved by using MATLAB bvp4c package. The obtained numerical results are presented through graphs (219) and Tabs 4-5. Also, we compare the present results with the existing results of Fang et al. [20] and Khader and Megahed [21] in Tabs 1-3 with some limiting cases. We fixed the value of non-dimensional parameters as $\operatorname{Pr}=0.71, M=2, K=2, A=0.5, R=0.5, N b=0.2, N t=0.3, \mathrm{Ec}=0.1, \eta=0.5, \mathrm{Sc}=2$, $n=0.3, b=0.5, \alpha=0.5, L_{1}=L_{2}=L_{3}=0.3$. The numerical values of the skin friction coefficient and the local Nusselt number for various flow quantities are presented in Tabs 4 and 5.

Table 1. Comparison of the numerical values of $f^{\prime \prime}(0)$ for $\alpha=0.5$ with $L_{1}=M=A=K=0$.

\begin{tabular}{|c|c|c|c|c|c|c|c|c|c|c|}
\hline$n$ & 10 & 9 & 7 & 5 & 3 & 2 & 1 & 0.5 & 0 & -0.5 \\
\hline Fang et al. [20] & -1.0603 & -1.0589 & -1.0550 & -1.0486 & -1.0359 & -1.0234 & -1.0000 & -0.9799 & -0.9576 & -1.1667 \\
\hline $\begin{array}{c}\text { Khader and } \\
\text { Megahed [21] }\end{array}$ & 1.0603 & 1.0588 & 1.0551 & 1.0486 & 1.0358 & 1.0234 & 1.0000 & 0.9798 & 0.9577 & 1.1666 \\
\hline Present result & 1.060343 & 1.058934 & 1.055063 & 1.048629 & 1.035884 & 1.023893 & 1.000484 & 0.981340 & 0.95803 & 1.167724 \\
\hline
\end{tabular}

Table 2. Comparison of the numerical values of $f^{\prime \prime}(0)$ for $\alpha=0.25$ with $L_{l}=M=A=K=0$.

\begin{tabular}{|c|c|c|c|c|c|c|c|c|c|c|}
\hline$n$ & 10 & 9 & 7 & 5 & 3 & 1 & 0.5 & 0 & $-1 / 3$ & -0.5 \\
\hline Fang et al. [20] & -1.1433 & -1.1404 & -1.1323 & -1.1189 & -1.0905 & -1.0000 & -0.9338 & -0.7843 & -0.5000 & -0.0833 \\
\hline $\begin{array}{c}\text { Khader and } \\
\text { Megahed [21] }\end{array}$ & 1.1433 & 1.1404 & 1.1322 & 1.1186 & 1.0904 & 1.0000 & 0.9337 & 0.7843 & 0.5000 & 0.0832 \\
\hline Present Result & 1.143334 & 1.140406 & 1.132299 & 1.118603 & 1.090508 & 1.000063 & 0.934325 & 0.78443 & 0.500417 & 0.086003 \\
\hline
\end{tabular}

Table 3. Comparison of the numerical values of $-f^{\prime \prime}(0)$ with $M=A=K=0$.

\begin{tabular}{|c|c|c|c|c|}
\hline$L_{1}$ & $\alpha$ & $n$ & Khader and Megahed [21] & Present Result \\
\hline 0.0 & 0.2 & 0.5 & 0.924828 & 0.925333 \\
\hline 0.2 & 0.2 & 0.5 & 0.728201 & 0.728725 \\
\hline 0.5 & 0.2 & 0.5 & 0.561082 & 0.561615 \\
\hline
\end{tabular}


Table 4. Numerical values of the skin friction coefficient when $\operatorname{Pr}=0.71, M=2, K=2, A=0.5$, $R=0.5, N b=0.2, N t=0.3, \mathrm{Ec}=0.1, \eta=0.5, \mathrm{Sc}=2, L_{1}=L_{2}=L_{3}=0.3, n=0.3, \alpha=0.5$.

\begin{tabular}{|c|c|c|c|}
\hline$M$ & $A$ & $\alpha$ & $C f_{x}$ \\
\hline 1 & & & 1.359824 \\
\hline 2 & & & 1.430758 \\
\hline 3 & & & 1.491775 \\
\hline & 0.1 & & 1.388477 \\
\hline & 0.3 & & 1.410127 \\
\hline & 0.5 & & 1.430758 \\
\hline & & 0.5 & 1.430758 \\
\hline & & 1.0 & 1.455985 \\
\hline & & 1.5 & 1.480581 \\
\hline
\end{tabular}

Table 5. Numerical values of the Nusselt number when $\operatorname{Pr}=0.71, M=2, \quad K=2, A=0.5$, $R=0.5, N b=0.2, N t=0.3, \mathrm{Ec}=0.1, \eta=0.5, \mathrm{Sc}=2, L_{1}=L_{2}=L_{3}=0.3, n=0.3, \alpha=0.5$.

\begin{tabular}{|c|c|c|c|c|c|c|c|c|}
\hline$K$ & $A$ & $R$ & $N b$ & $N t$ & $\mathrm{Ec}$ & $\alpha$ & $\mathrm{Sc}$ & $\mathrm{Nu}_{x}$ \\
\hline 1 & & & & & & & & 0.250578 \\
\hline 2 & & & & & & & & 0.237039 \\
\hline 3 & & & & & & & & 0.225986 \\
\hline & 0.1 & & & & & & & 0.282042 \\
\hline & 0.3 & & & & & & & 0.259321 \\
\hline & 0.5 & & & & & & & 0.237039 \\
\hline & & 1 & & & & & & 0.223307 \\
\hline & & 2 & & & & & & 0.210681 \\
\hline & & 3 & & & & & & 0.204781 \\
\hline & & & 0.1 & & & & & 0.249088 \\
\hline & & & 0.3 & & & & & 0.225419 \\
\hline & & & 0.5 & & & & & 0.203444 \\
\hline & & & & 0.1 & & & & 0.246615 \\
\hline & & & & 0.3 & & & & 0.237039 \\
\hline & & & & 0.5 & & & & 0.228055 \\
\hline & & & & & 0.1 & & & 0.237039 \\
\hline & & & & & 0.5 & & & 0.177254 \\
\hline & & & & & 0.7 & & & 0.157452 \\
\hline & & & & & & 0.5 & & 0.237039 \\
\hline & & & & & & 1.0 & & 0.268861 \\
\hline & & & & & & 1.5 & & 0.300584 \\
\hline & & & & & & & 0.2 & 0.209707 \\
\hline & & & & & & & 0.4 & 0.215470 \\
\hline & & & & & & & 0.6 & 0.220107 \\
\hline
\end{tabular}

Figure 2 illustrates the influence of the magnetic field parameter $(M)$ on the velocity profile and shows that the fluid velocity decreases with increasing of $M$. This is due to the existence of magnetic field sets a resistive force called Lorentz force, which behaves as a retarding force on the fluid velocity and consequently reduces the fluid flow. The impact of the porosity parameter $(K)$ on velocity profiles $f^{\prime}(\eta)$ and $g^{\prime}(\eta)$ is shown in Fig.3. It is 
observed that for increasing values of $K$ the fluid velocity decreases. The porous medium imposes high limitations on the fluid flow and slows down the fluid motion. Therefore, on increasing the permeability parameter the resistance of the fluid motion increases and hence velocity decreases. Figures 4-6 display the influence of the power law index parameter $(n)$ on the axial velocity $\left(f^{\prime}(\eta)\right)$, transverse velocity $\left(g^{\prime}(\eta)\right)$, temperature and concentration fields. It is seen that the velocity, temperature and concentration profiles are increased with increasing the value of $n$ and also a significant change is observed in the fluid characteristics for a shear thickening fluid $(n>1)$ than that of a shear thinning fluid $(n<1)$. Generally, a thin surface generates more heat than a thick surface. Therefore, enhancing the velocity power law index parameter tends to reduce the thickness of the sheet. Therefore the temperature of the fluid increases.

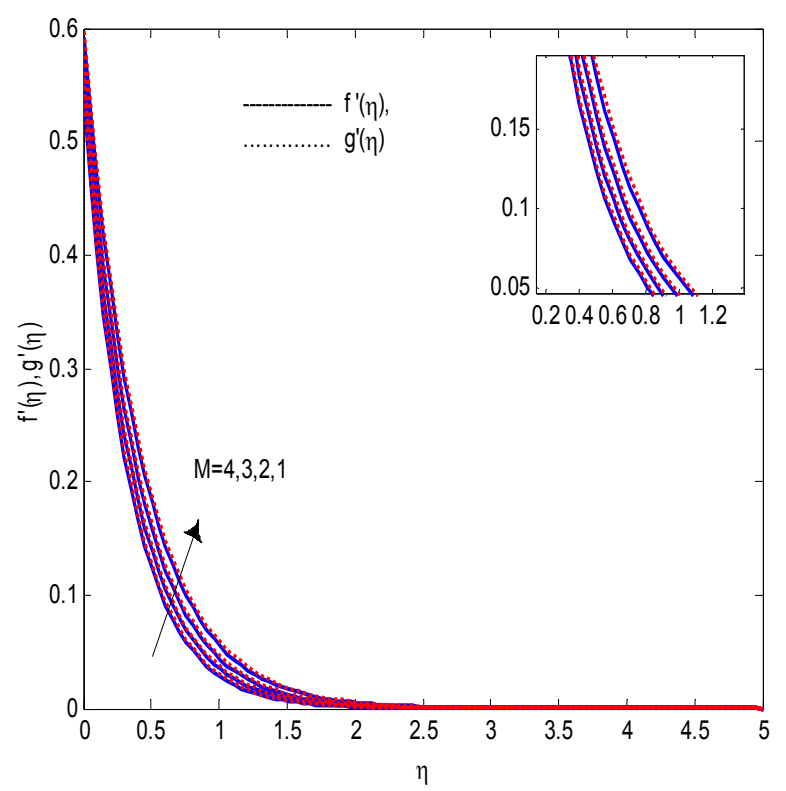

Fig.2. Effect of $M$ on $f^{\prime}(\eta)$ and $g^{\prime}(\eta)$.

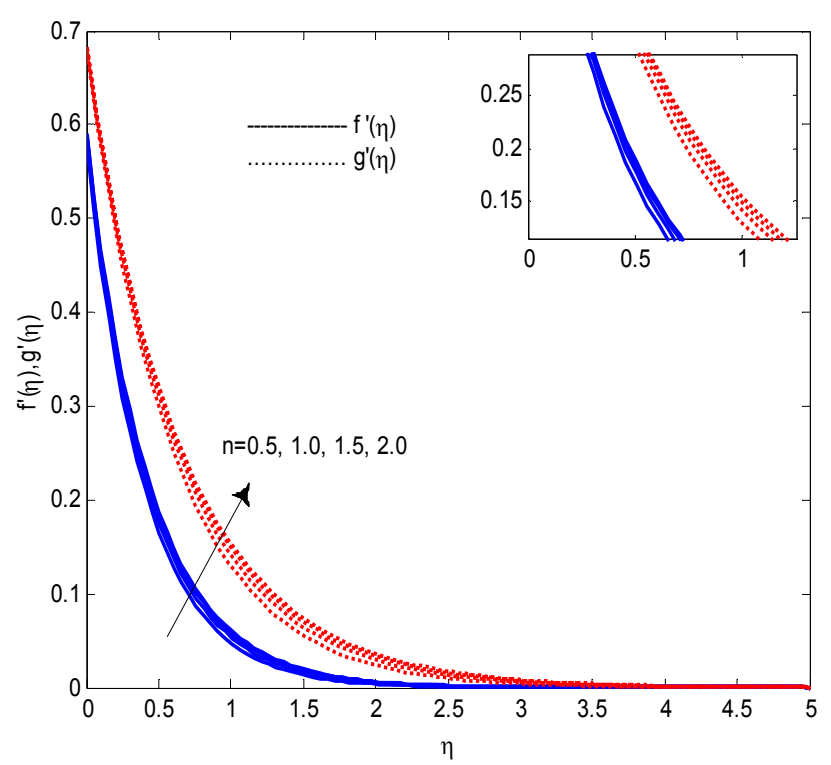

Fig.4. Effect of $n$ on $f^{\prime}(\eta)$ and $g^{\prime}(\eta)$.

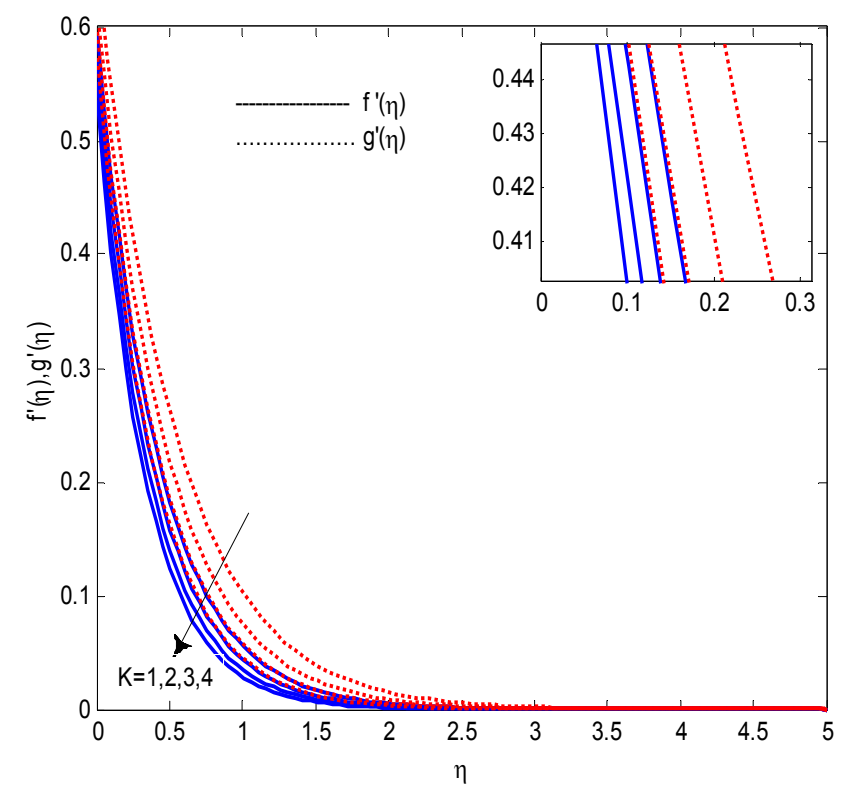

Fig.3. Effect of $K$ on $f^{\prime}(\eta)$ and $g^{\prime}(\eta)$.

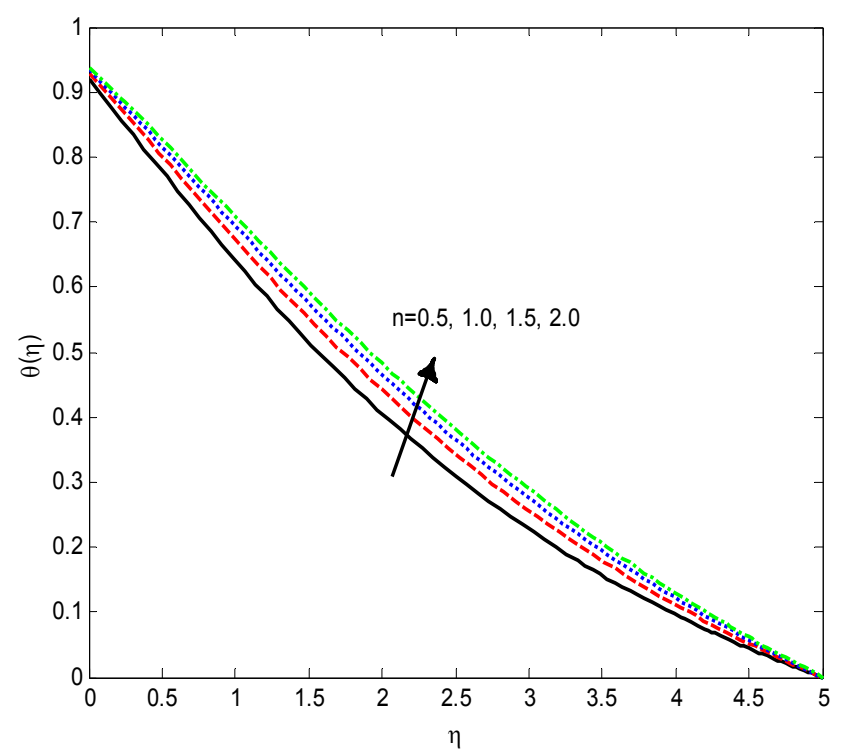

Fig.5. Effect of $n$ on $\theta(\eta)$. 


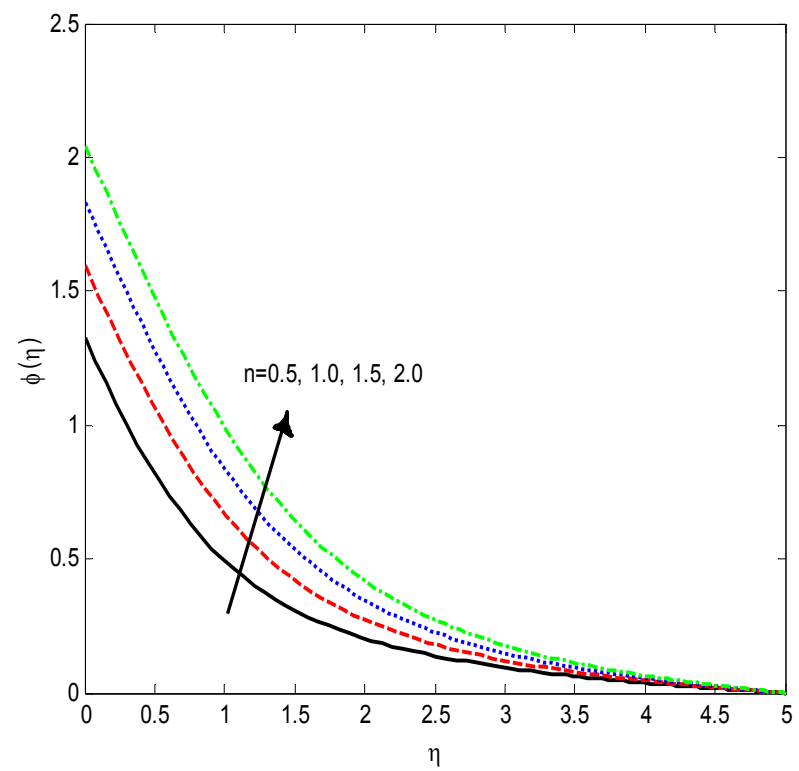

Fig.6. Effect of $n$ on $\phi(\eta)$.

Figure 7 presents the effect of the thermal radiation parameter on the temperature field. From the figure it is clear that the fluid temperature increased with increasing values of $(R)$. The velocity profiles for various values of the unsteady parameter $(A)$ are shown in Fig.8. It is observed that the velocity profiles are decreased with an increase in $(A)$. An opposite trend is observed on temperature profiles (see Fig.9). Figures 10-12 show the velocity, temperature and concentration profiles with the impact of the variable wall thickness parameter $(\alpha)$. It is evident that for large values of $\alpha$, there is a smaller deformation in the stretching of wall and hence the fluid velocity decreases. As said above, thick surfaces slowly emit the heat into the flow. Therefore, increasing the value of $\alpha$ is to reduce the heat dissipation towards the flow and reduce the temperature profiles. A similar phenomenon is observed on the concentration profile.

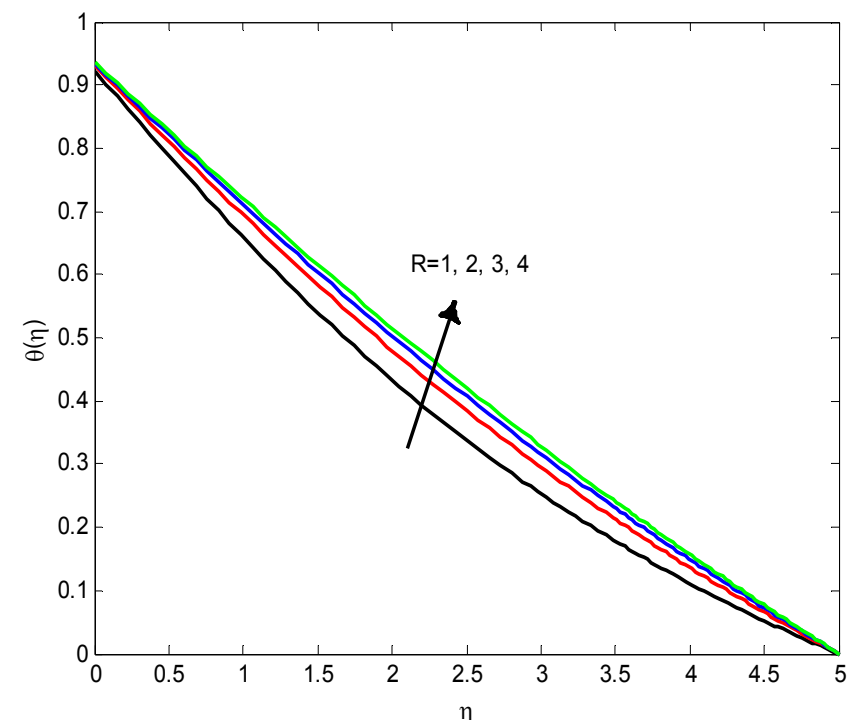

Fig.7. Effect of $R$ on $\theta(\eta)$.

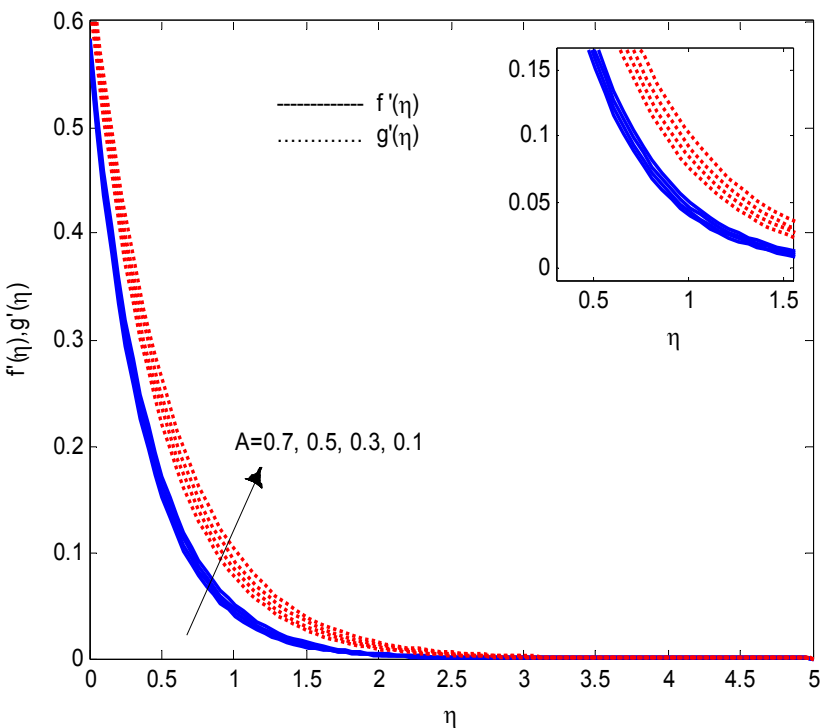

Fig.8. Effect of $A$ on $f^{\prime}(\eta)$ and $g^{\prime}(\eta)$. 


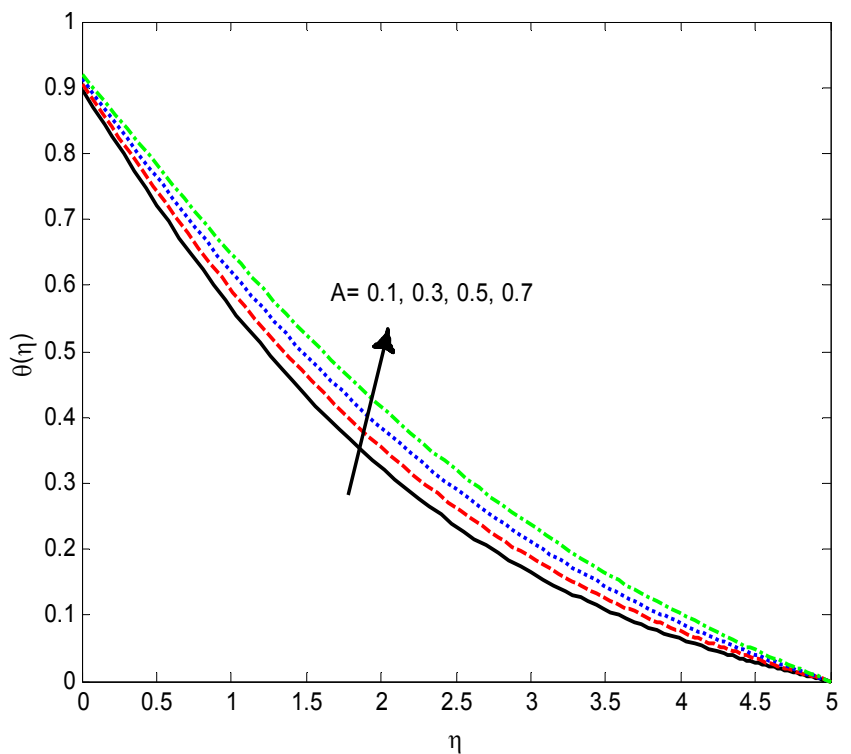

Fig.9. Effect of $A$ on $\theta(\eta)$.

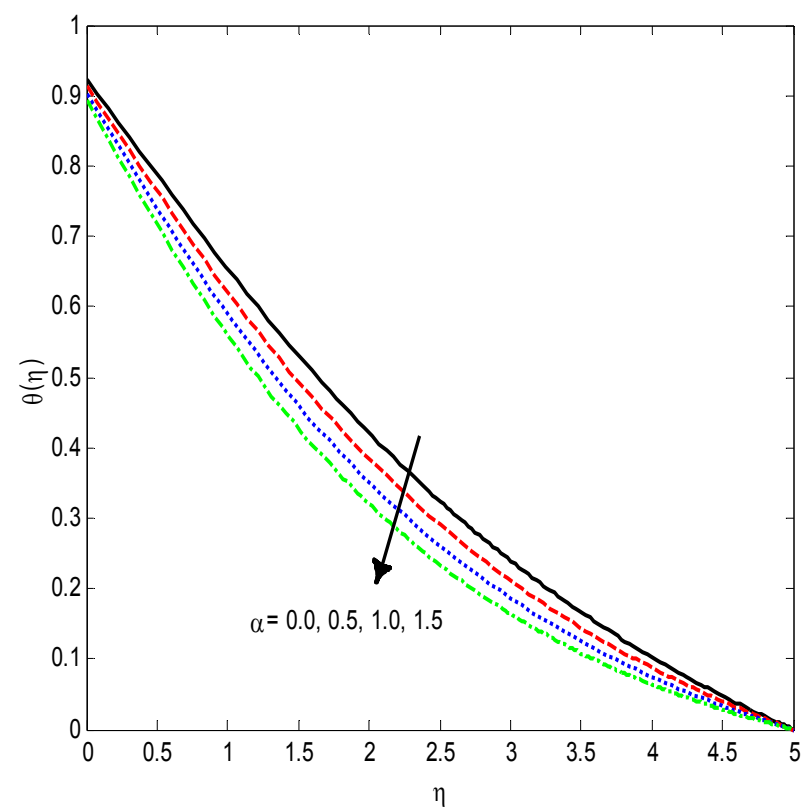

Fig.11. Effect of $\alpha$ on $\theta(\eta)$.

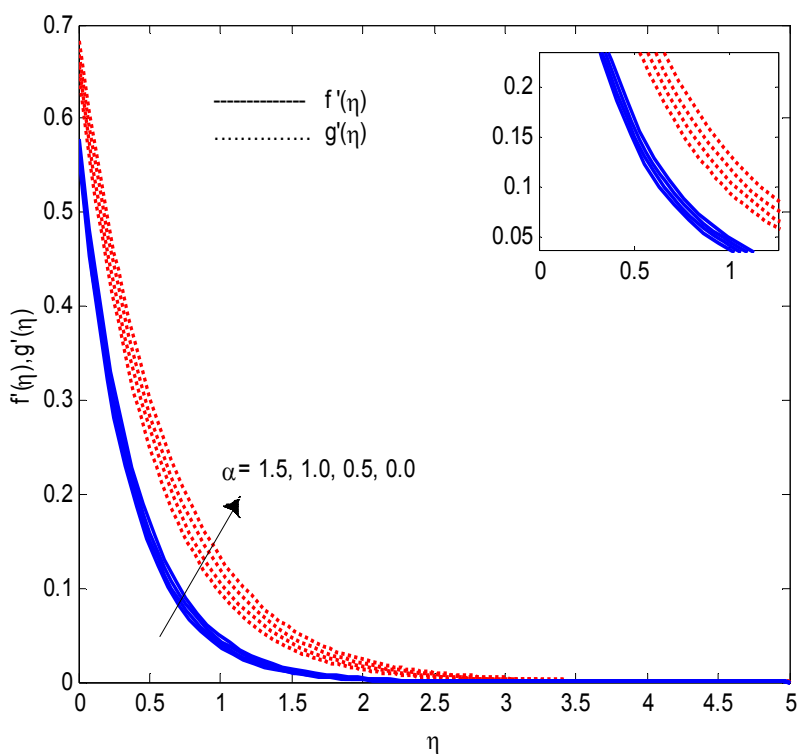

Fig.10. Effect of $\alpha$ on $f^{\prime}(\eta), g^{\prime}(\eta)$.

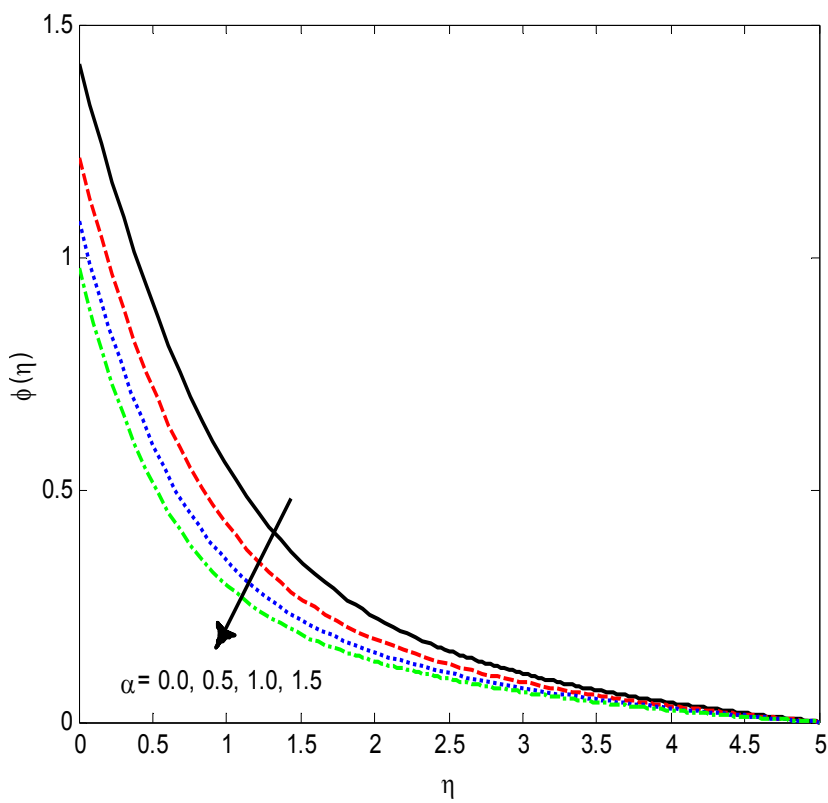

Fig.12. Effect of $\alpha$ on $\phi(\eta)$.

Figures 13 and 14 illustrate the impact of thermophoresis $(N t)$ on temperature and concentration fields. It is noted that on increasing the values of $N t$ the temperature and concentration profiles of a fluid increase significantly. This is due to suspension of micro sized particles into fluid acquire an increment in the temperature difference between the fluid and the sheet. In accordance with this, the thermal boundary layer thickness increases and hence temperature increases. A similar trend is observed on concentration profiles. Because for higher values of $N t$, the thermophoretic difference becomes stronger and absorbs the particles from the hotter region to the cooler region. In fact, the Brownian motion tries to push the particles in the direction opposite to the concentration gradient and makes the nanofluid more homogeneous. Hence, for higher values of $(\mathrm{Nb})$ the concentration becomes low (see Fig.15). 


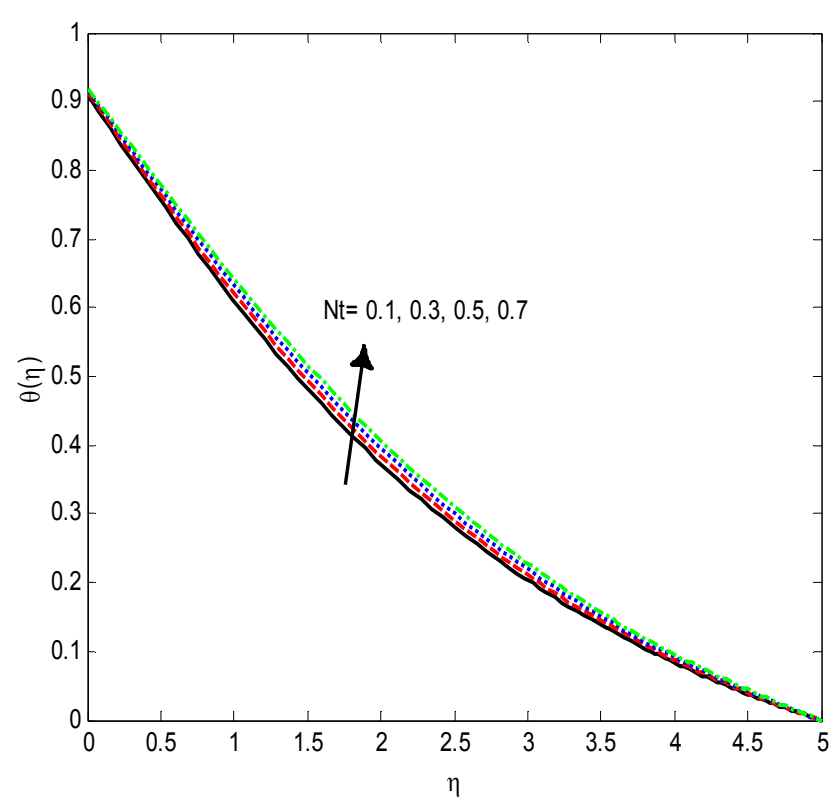

Fig.13. Effect of $N t$ on $\theta(\eta)$.

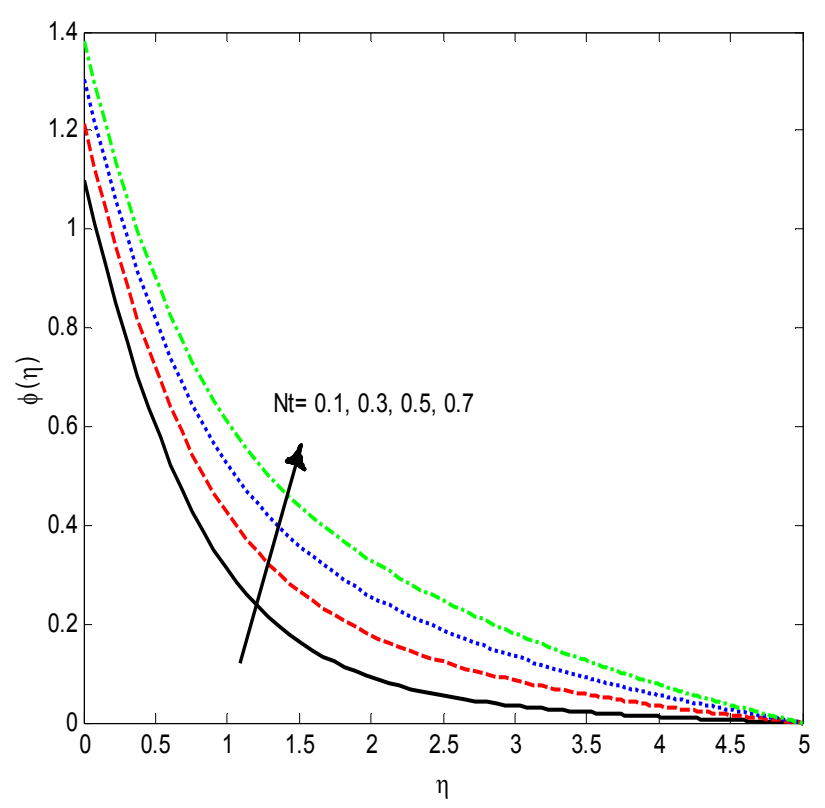

Fig.14. Effect of $N t$ on $\phi(\eta)$.

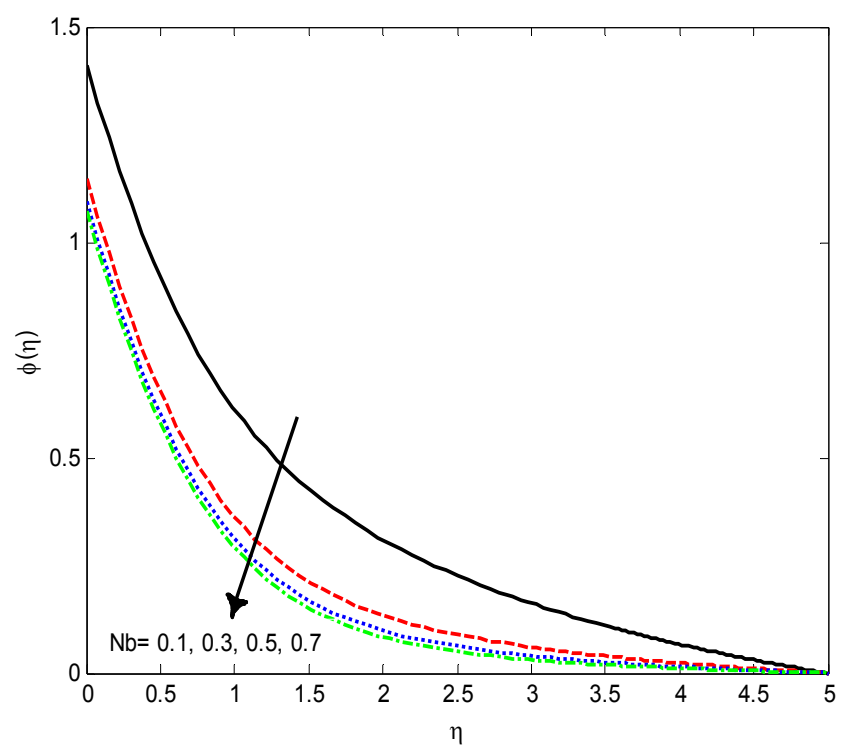

Fig.15. Effect of $N b$ on $\phi(\eta)$.

Figure 16 shows the influence of the velocity slip parameter $\left(L_{l}\right)$ on $f^{\prime}(\eta)$ and $g^{\prime}(\eta)$. It is seen that the velocity profiles decrease with increasing values of $L_{l}$. The fluid temperature decreases with the thermal slip parameter. This is because an increasing slip parameter causes to reduce the heat transfer phenomenon. Therefore, the temperature of the fluid decreases (see Fig.17). Figure 18 presents the concentration profiles for the solutal slip parameter $\left(L_{3}\right)$. It is clear that the increasing values of $L_{3}$ decrease the concentration profiles of the fluid. The effect of the Eckert number (Ec) on the temperature profile is shown in Fig.19. It is seen that the fluid temperature increases in the presence and absence of the radiation parameter $(R)$ with an increase value of Eckert number (Ec). 
Physically, the Eckert number is the relation between the flow kinetic energy and heat enthalpy difference. Therefore, Ec improves the kinetic energy and consequently increases the temperature of a fluid. Further, we noticed that there is more significant in temperature profiles when $R>0$, than $R=0$.

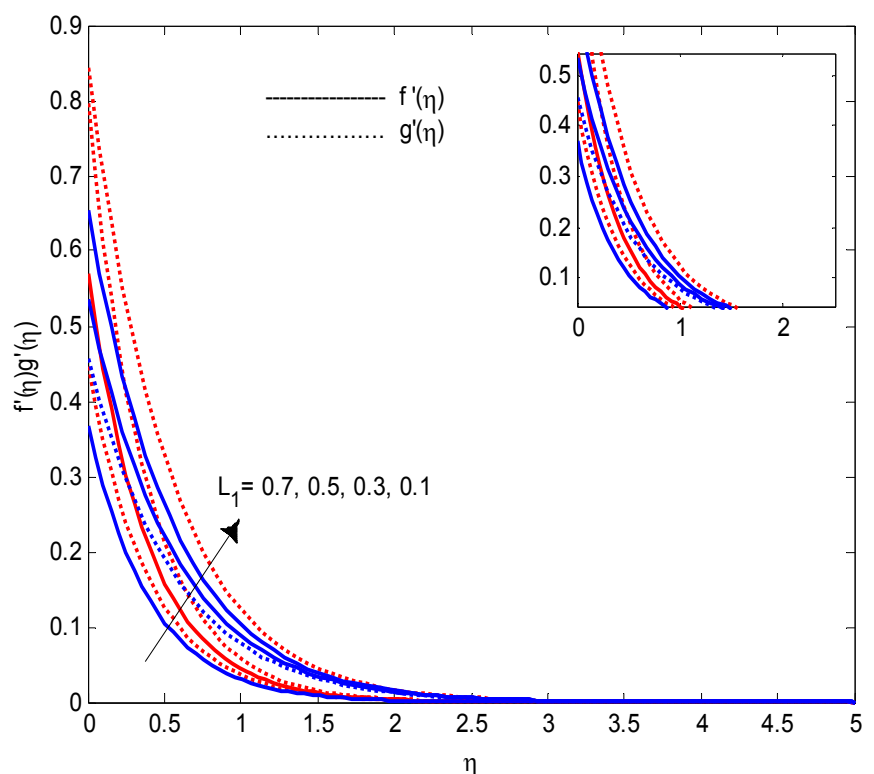

Fig.16. Effect of $L_{l}$ on $f^{\prime}(\eta)$ and $g^{\prime}(\eta)$.

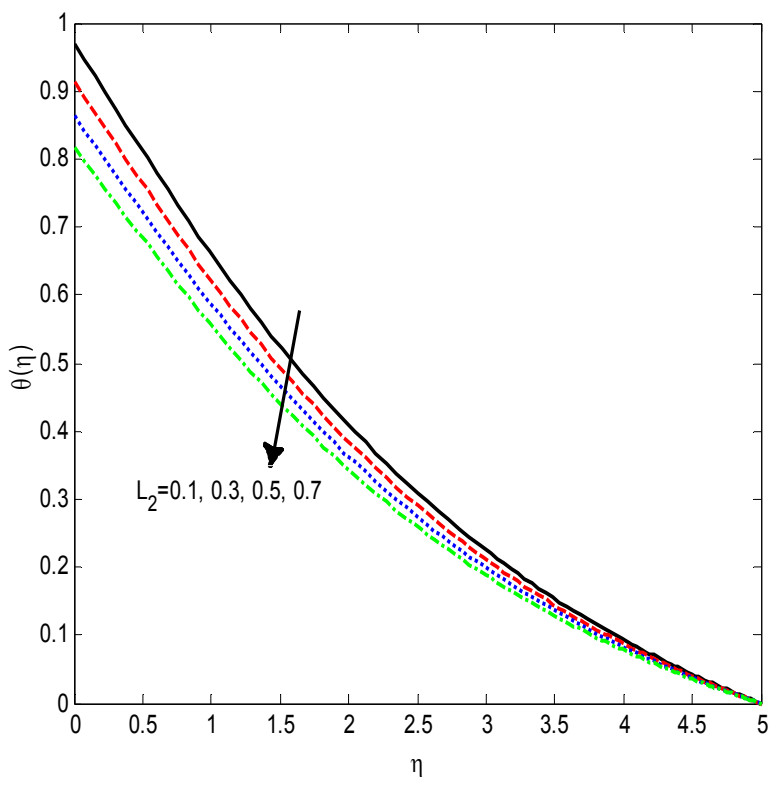

Fig.17. Effect of $L_{2}$ on $\theta(\eta)$.

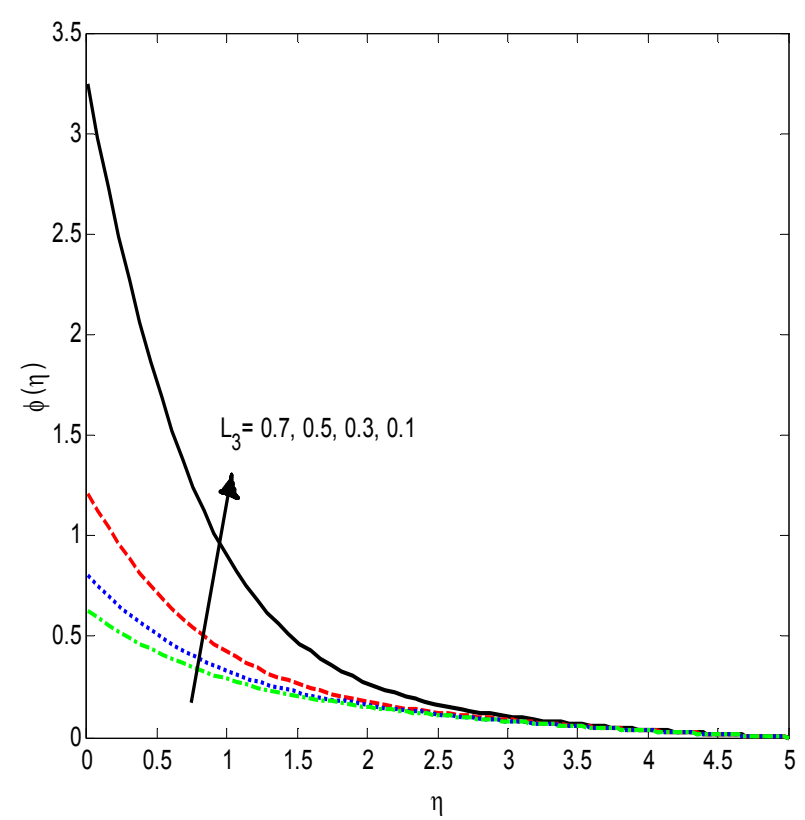

Fig.18. Effect of $L_{3}$ on $\phi(\eta)$.

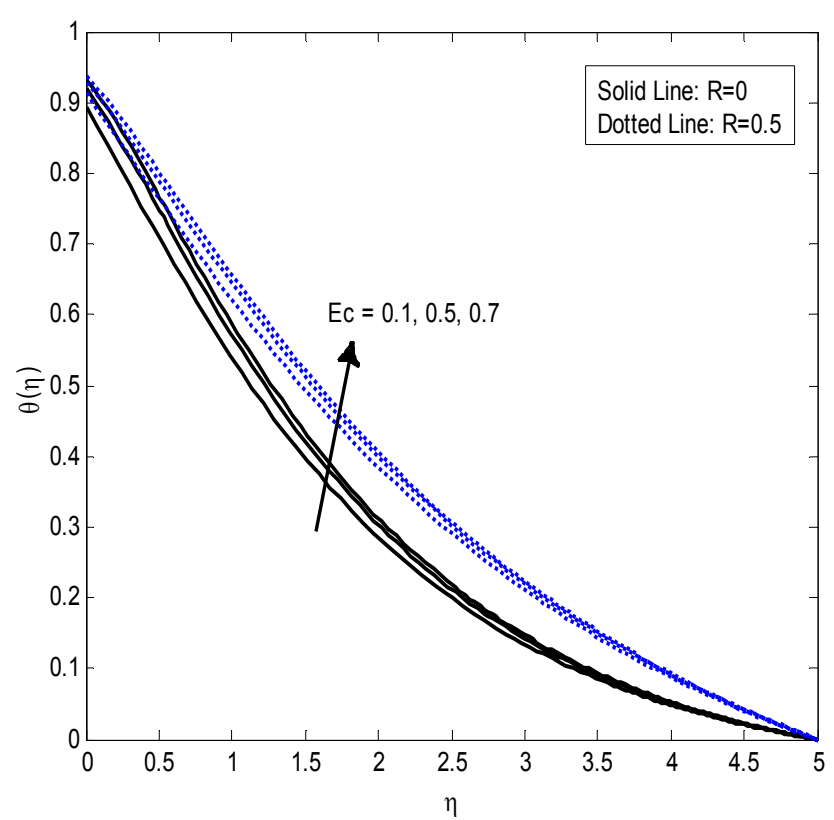

Fig.19. Effect of Ec on $\theta(\eta)$.

Tables 1-3 show the comparison of present results with the existing results of Fang et al. [20] and Khader et al. [21]. In the absence of certain parameters the present results are in good agreement with the existing results. The numerical values of the skin friction coefficient and the rate of heat transfer coefficient are presented in Tabs 4 and 5. From Tab.4 it is clear that the friction factor coefficient increases with $M, A$ and $\alpha$. From this result it is concluded that the magnetic field, unsteady parameter and wall thickness 
parameters increase the fluid flow. From Tab.5, it is seen that the heat transfer rate is decreased for higher values of $A, R, N t, N b, \mathrm{Ec}$, while a quite opposite trend is observed for $\alpha$ and $\mathrm{Sc}$.

\section{Final remarks}

In the present work we investigate the unsteady three dimensional nanofluid flow over an exponentially stretching sheet in the presence of slip conditions. The important remarks which can be found in the present study are:

1. High values of the magnetic field parameter increase the skin friction coefficient.

2. An increase in the Eckert number leads to more heat transfer in the fluid.

3. The Nusselt number and Sherwood numbers decrease for growing values of the thermophoretic parameter.

4. More heat is transferred with growing values of the unsteady parameter.

5. There is a significant change in the velocity, temperature and concentration profiles for a shear thickening fluid than for a shear thinning fluid with the impact of the power law index parameter.

\section{Nomenclature}

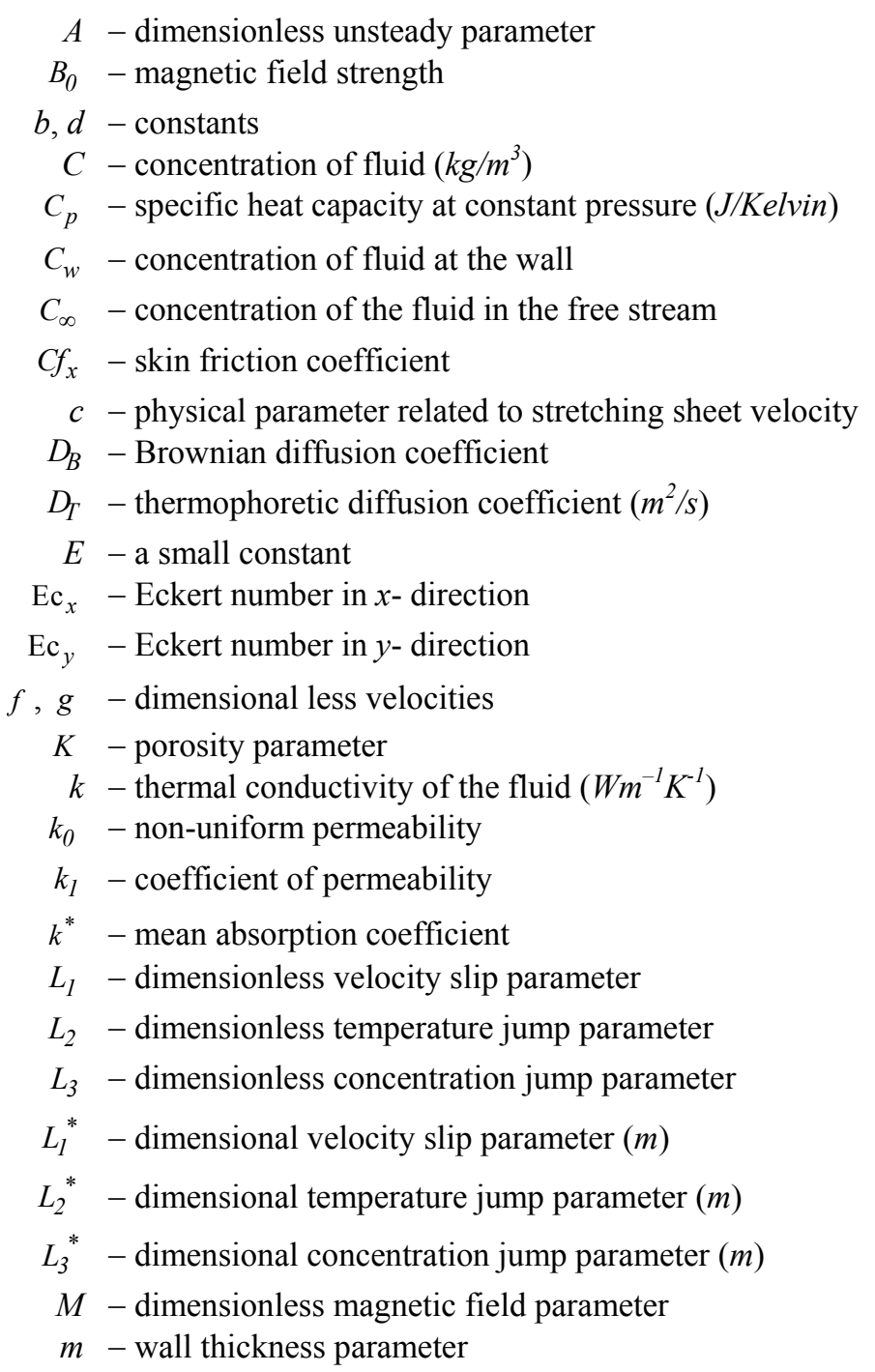




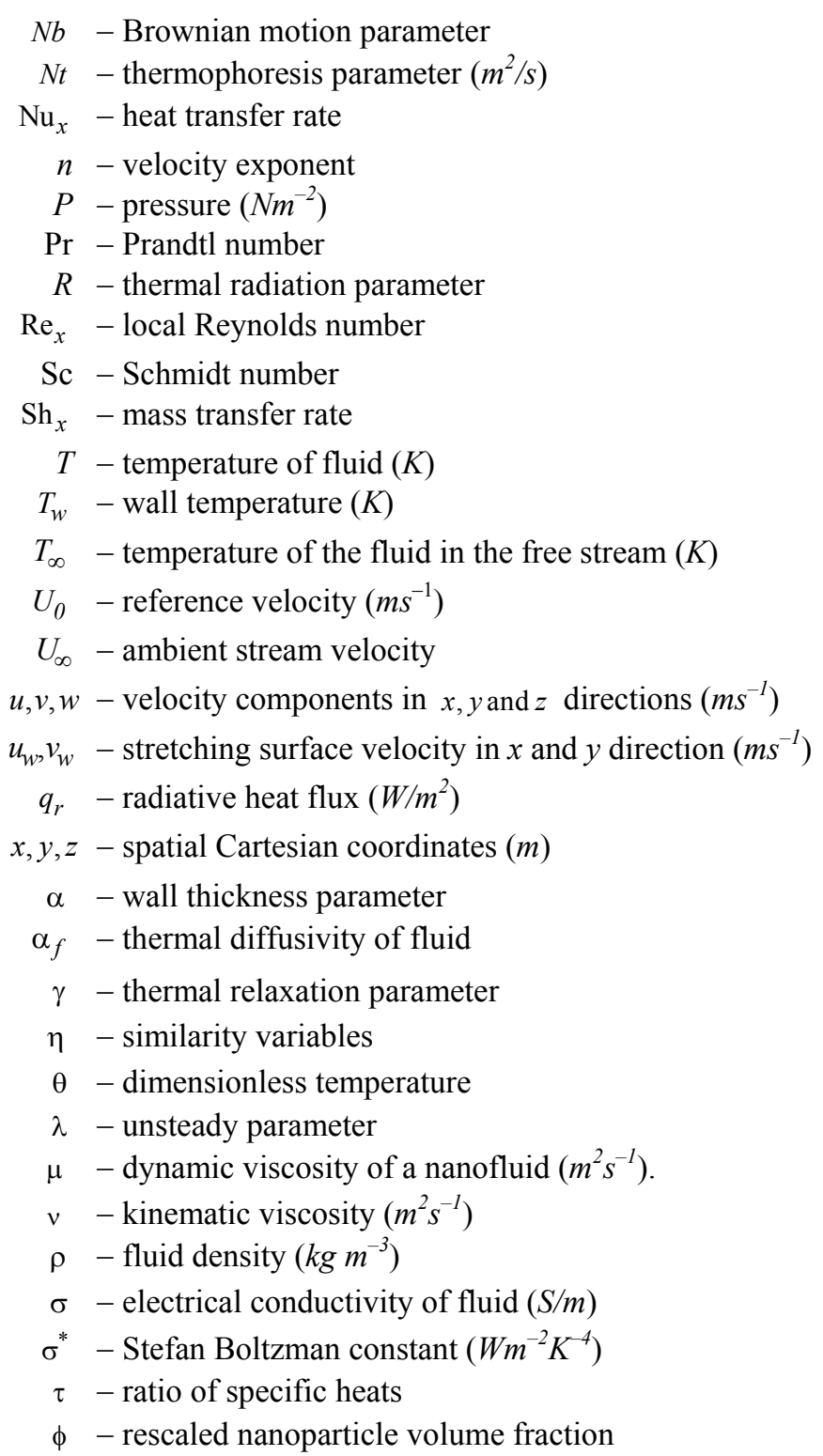

\section{Subscripts}
$f \quad$ - base fluid
$w$ - condition at the wall
$\infty$ - ambient condition

\section{Superscripts}

' - differentiation with respect to $\eta$

* - dimensional properties

\section{References}

[1] Crane L.J. (1970): Flow past a stretching plate. - Z. Angew. Math. Phys, vol.21, pp.645-647. 
[2] Khan W.A. and Pop I. (2010): Boundary-layer flow of a nanofluid past a stretching sheet. - International Journal of Heat and Mass Transfer, vol.53, pp.2477-2483.

[3] Nadeemand S. and Lee C. (2012): Boundary layer flow of nanofluid over an exponentially stretching surface. Nanoscale Research Letters, vol.94, pp.1-6.

[4] Mansur S. and Ishak A. (2016): Unsteady boundary layer flow of a nanofluid over a stretching/shrinking sheet with a convective boundary condition. - Journal of the Egyptian Mathematical Society, vol.24, pp.650-655.

[5] Mabood F., Khan W.A. and Ismail A.I.M. (2015): MHD boundary layer flow and heat transfer of nanofluids over a nonlinear stretching sheet: A numerical study. - Journal of Magnetism and Magnetic Materials, vol.374, pp.569576.

[6] Bhattacharyya K. and Layek G.C. (2014): Magnetohydrodynamic boundary layer flow of nanofluid over an exponentially stretching permeable sheet. - Hindawi Publishing Corporation Physics Research International, vol.2014, pp.1-12.

[7] Daba M. and Devaraj P. (2016): Unsteady boundary layer flow of a nanofluid over a stretching sheet with variable fluid properties in the presence of thermal radiation. - Thermophysics and Aeromechanics, vol.23, pp.403-413.

[8] Sharma R., Ishak A. and Pop I. (2013): Partial slip flow and heat transfer over a stretching sheet in a nanofluid. Hindawi Publishing Corporation, Mathematical Problems in Engineering, vol.2013, pp.1-7.

[9] Maxwell J.C. (1879): On stresses in rarefied gases arising from inequalities of temperature. - Philosophical Transactions: The Royal Society London, vol.170, pp.231-256.

[10] Hak G.M. (2002): Flow Physics. - In: Gad-el-Hak, M (ed.) The MEMS Handbook. CRC Press, Boca Raton.

[11] Beavers G.S. and Joseph D.D. (1967): Boundary condition at a naturally permeable wall. - Journal of Fluid Mechanics, vol.30, pp.197-207.

[12] Das K. (2015): Nanofluid flow over a non-linear permeable stretching sheet with partial slip. - Journal of the Egyptian Mathematical Society, vol.23, pp.451-456.

[13] Goyal M. and Bhargava R. (2014): Boundary layer flow and heat transfer of viscoelastic nanofluids past a stretching sheet with partial slip conditions. - Applied Nanoscience, vol.4, pp.761-767.

[14] Awais M., Hayat T., Ali A. and Irum S. (2016): Velocity, thermal and concentration slip effects on a magnetohydrodynamic nanofluid flow. - Alexandria Engineering Journal, vol.55, pp.2107-2114.

[15] Raza J., Mohd Rohni A., Omar Z. and Awais M. (2016): Heat and mass transfer analysis of MHD nanofluid flow in a rotating channel with slip effects. - Journal of Molecular Liquids, vol.219, pp.703-708.

[16] Ibrahim W. and Shankar B. (2013): MHD boundary layer flow and heat transfer of a nanofluid past a permeable stretching sheet with velocity, thermal and solutal slip boundary conditions. - Computers and Fluids, vol.75, pp. $1-10$.

[17] Nagendramma V., Kiran Kumar R.V.M.S.S., Durga Prasad P., Leelaratnam A. and Varma S.V.K. (2016): Multiple slips and radiation effects on Maxwell nanofluid flow over a permeable stretching surface with dissipation. Journal of Nanofluids, vol.5, pp.817-825.

[18] Kiran Kumar R.V.M.S.S. and Varma S.V.K. (2017): Multiple slips and thermal radiation effects on MHD boundary layer flow of a nanofluid through porous medium over a nonlinear permeable sheet with heat source and chemical reaction. - Journal of Nanofluids, vol.6, pp.48-58.

[19] Shaw S., Kameswaran P.K. and Sibanda P. (2016): Effects of slip on nonlinear convection in nanofluid flow on stretching surfaces. - Boundary Value Problems, vol.2, pp.1-11.

[20] Fang T., Zhang J. and Yongfang Zhong (2012): Boundary layer flow over a stretching sheet with variable thickness. - Applied Mathematics and Computation, vol.218, pp.7241-7252.

[21] Khader M.M. and Ahmed M. Megahed (2013): Numerical solution for boundary layer flow due to a nonlinearly stretching sheet with variable thickness and slip velocity. - European Physics Journal Plus, vol.128, 100. 
[22] Anjali Devi S.P. and Prakash M. (2015): Temperature dependent viscosity and thermal conductivity effects on hydromagnetic flow over a slendering stretching sheet. - Journal of the Nigerian Mathematical Society, vol.34, pp.318-330.

[23] Anjali Devi S.P. and Prakash M. (2016): Thermal radiation effects on hydromagnetic flow over a slendering stretching sheet. - Journal of Brazilian Society Mechanical Science and Engineering, vol.38, pp.423-431.

[24] Abdel-Wahed M.S., Elbashbeshy E.M.A. and Emam T.G. (2015): Flow and heat transfer over a moving surface with non-linear velocity and variable thickness in a nanofluids in the presence of Brownian motion. - Applied Mathematics and Computation, vol.254, pp.49-62.

[25] Kiran Kumar R.V.M.S.S. and Varma S.V.K. (2017): Hydromagnetic boundary layer slip flow of nanofluid through porous medium over a slendering stretching sheet. - Journal of Nanofluids, vol.6, pp.852-861.

[26] Kiran Kumar R.V.M.S.S., Vijaya Kumar Varma S., Raju C.S.K., Ibrahim S.M., Lorenzini G. and Lorenzini E. (2017): Magnetohydrodynamic 3D slip flow in a suspension of carbon nanotubes over a slendering sheet with heat source/sink. - Continuum Mechanics and Thermodynamics, vol.29, pp.835-851.

[27] Hayat T., Waqas M., Alsaedi A., Bashir G. and Alzahrani F. (2017): Magnetohydrodynamic (MHD) stretched flow of tangent hyperbolic nanoliquid with variable thickness. - Journal of Molecular Liquids, vol.229, pp.178-184.

[28] Acharya N., Das K. and Kumar Kundu P. (2016): Ramification of variable thickness on MHD TiO2 and Ag nanofluid flow over a slendering stretching sheet using NDM. - European Physics Journal Plus, vol.131, No.303, pp.1-16.

[29] Prasad K.V., Vajravelu K., Vaidya H. and Robert A. Van Gorder (2017): MHD flow and heat transfer in a nanofluid over a slender elastic sheet with variable thickness. - Results in Physics, vol.7, pp.1462-1474.

Received: December 2, 2017

Revised: August 11, 2018 